\title{
Molecular cloning and sequence analysis of follicle-stimulating hormone beta polypeptide precursor cDNA from the bovine pituitary gland
}

\author{
L.S. Dai ${ }^{1,2}$, Y.M. Zhao ${ }^{3}$, G.L. Zhang ${ }^{3}$, R.F. Zhao ${ }^{1,4}$, H. Jiang ${ }^{1,2}$, T.H. Ma $^{1,2}$, \\ Y. Gao ${ }^{1,2}$, B. Yuan ${ }^{1,2}$, Y.L. Xu ${ }^{1,2}$, W.Y. Yu ${ }^{1,2}$, Z.H. Zhao ${ }^{2}$ and J.B. Zhang ${ }^{1}$ \\ ${ }^{1}$ Laboratory Animal Center, Jilin University, Changchun, Jilin, China \\ ${ }^{2}$ College of Animal Science and Veterinary Medicine, \\ Jilin University, Changchun, Jilin, China \\ ${ }^{3}$ Jilin Academy of Agricultural Sciences, Changchun, Jilin, China \\ ${ }^{4}$ Breeding Center of High-Quality Cattle, Changchun, Jilin, China \\ Corresponding author: J.B. Zhang or Z.H. Zhao \\ E-mail: zjb515@163.com / zhzhao@jlu.edu.cn
}

Genet. Mol. Res. 10 (3): 1504-1513 (2011)

Received August 2, 2010

Accepted January 28, 2011

Published July 25, 2011

DOI 10.4238/vol10-3gmr997

\begin{abstract}
Follicle-stimulating hormone (FSH) plays an essential role in mammalian spermatogenesis and follicular development. In a previous study, we demonstrated that some bulls carry numerous linked mutations in the FSH beta-subunit $(F S H B)$ gene, and that these bulls have poorquality semen, low fertility, and slightly lower serum FSH concentration compared to those without such mutations. Here, we identified the different $F S H B$ mRNA transcripts in such individuals and analyzed the evolutionary pattern of the $F S H B$ open reading frame (ORF) in different species. Two different lengths of $F S H B$ mRNA transcripts corresponding to two different polyadenylation sites in the 3'-UTR were detected in wild-type bull pituitary glands, and four different mRNA transcripts resulting from the different polyadenylation sites and linked mutations were identified in mutation-bearing bull pituitaries. All transcripts had almost the same putative $F S H B$ precursor molecule. When the ORF
\end{abstract}


sequences of wild-type and mutation-bearing genes were compared with those of other tetrapod species, the leopard frog had the lowest level of homology (57.8 and 58.1\%) and the buffalo had the highest level (95.9 and $96.7 \%$ ), respectively. These results indicated that the bovine FSHB gene transcribes at least two classes of mRNA in the wild-type and four classes of mRNA in the mutation-bearing individuals, which provides a new insight into the bovine $F S H B$ evolutionary pattern. In addition, these findings lay a foundation for further study of gene expression regulation and the effects of mutations on male fertility traits in cattle.

Key words: Bovine; FSHB; RACE; mRNA transcripts

\section{INTRODUCTION}

Follicle-stimulating hormone (FSH), glycoprotein hormone derived from adenohypophyseal parenchymal cells, is a key regulator of the reproductive process in mammals. It is involved in the initiation and early stages of spermatogenesis through binding to FSH receptors in males, and follicular development and maturation in females (Dias et al., 2002; de Kretser et al., 2004; Kumar, 2005). Consistent with other members of the glycoprotein hormone family (i.e., luteinizing hormone, thyroid-stimulating hormone, and chorionic gonadotropin), functional FSH is a heterodimer formed by an $\alpha$-subunit shared with other glycoprotein hormones and a specific $\beta$-subunit encoded by the FSHB gene (Pierce and Parsons, 1981; Gharib et al., 1990).

The cDNA sequences encoding $F S H B$ polypeptide precursors have been cloned and characterized in most vertebrates, revealing that $F S H B$ mRNA has a longer 3' untranslated region (UTR), which is absent in other members of the glycoprotein family. Four distinct FSHB mRNA transcripts were found in humans, resulting from one alternate splicing donor site in exon 1 and two different polyadenylation sites in exon 3 (Jameson et al., 1988). Three different lengths of $F S H B$ mRNA transcript were found in rabbits as a result of the polyadenylation variants of one large transcript (Noguchi et al., 2006). However, it is still not clear whether there are alternative splicing and polyadenylation sites in the bovine FSHB mRNA transcript.

So far, some QTL associated with sperm quality were detected in bulls (Druet et al., 2009) and boars (Ren et al., 2009; Xing et al., 2009), and also some candidate genes are considered to be associated with male animal fertility traits, such as swine actin (Wimmers et al., 2005; Lin et al., 2006) and murine Сapza3 (Geyer et al., 2009). Our previous findings showed that some bulls with numerous linked mutations in the FSHB gene, including 13 substitutions and 1 insertion in the upstream regulation region (5'-URR) and the coding region of exon 3 , exhibited slightly lower serum FSH concentration, poor-quality semen, and low fertility (Dai et al., 2009). Therefore, it is very probable that different FSHB transcripts exist in such particular individuals so that different expression levels of the FSHB polypeptide precursor exist, which further affects the FSH concentration in the serum.

For the reasons above, we report here the cloning and sequence analysis of the bovine FSHB mRNA transcripts from the bulls carrying FSHB mutations or not, which provides not only the necessary information for understanding the $F S H B$ evolutionary pattern but also a useful tool for further study on gene expression regulation and elucidation of molecular mechanisms of $F S H B$ mutation effects on male reproduction traits. 


\section{MATERIAL AND METHODS}

\section{Sampling}

The five bulls with the linked mutations (four Simmental and one Charolais) have all been eliminated due to lower semen quality and fertility in our previous study population. Two pituitary glands belonging to these particular mutation-bearing individuals (one Simmental and one Charolais) were obtained from the local slaughter house, and another pituitary gland was from a wild-type individual (Simmental breed) to be used as a control. The tissues were frozen in liquid nitrogen immediately after dissection, and then stored at $-80^{\circ} \mathrm{C}$ until analysis.

\section{Preparation of RNA}

Total RNA was isolated using TRIzol Reagent (Invitrogen, CA, USA) according to the standard protocol, and dissolved in $0.1 \%$ DEPC-treated water. RNA quality was checked by electrophoresis using an ethidium bromide-stained agarose gel, and RNA concentrations were determined with a UV-2802H spectrophotometer (Unico, Shanghai Optical Company, China) at $260 \mathrm{~nm}$. RNA samples with $A_{260 \mathrm{~nm}} / A_{280 \mathrm{~mm}}$ ratios of 1.8 to 2.0 were used for further analysis.

\section{Amplification of full-length cDNA}

Full-length sequences of FSHB were obtained using a combination of 5'- and 3'-rapid amplification of cDNA ends (RACE). For 5'-RACE, we used a 5'-RACE cDNA amplification kit (Invitrogen) and primers GSP1 (5'-GCTGCATTTAGGACTTTC-3'), GSP2 (5'-AGCACCC TCGTGTCTGTAAGTTAAG-3'), and GSP3 (5'-TCAGGAGAGCAGAGATCAGTAGGGT-3'), based on the published mRNA sequences (GenBank No. M14853; Maurer and Beck, 1986). The locations of primers are shown in Figure 2. The first-strand cDNA was synthesized at $42^{\circ} \mathrm{C}$ for 50 min with $3 \mu \mathrm{g}$ total RNA using the GSP1 primer and SuperScript ${ }^{\mathrm{TM}}$ II reverse transcriptase (Invitrogen), and tailed with dCTP using terminal deoxynucleotidyl transferase. Two consecutive polymerase chain reactions (PCR) using this cDNA as a template and Ex Taq polymerase (Takara, Dalian, China) were carried out as follows: the first PCRs were performed for 32 cycles at $94^{\circ} \mathrm{C}$ for $30 \mathrm{~s}, 62^{\circ} \mathrm{C}$ for $30 \mathrm{~s}$, and $72^{\circ} \mathrm{C}$ for $1 \mathrm{~min}$ in a typical PCR mixture $(50 \mu \mathrm{L})$ with GSP2 primer and 5'-RACE abridged anchor primer (AAP) (5'-GGC CACGCGTCGACTAGTACGGGIIGGGIIGGGIIG-3'). The initial PCR product was diluted 1:20 with $\mathrm{ddH}_{2} \mathrm{O}$ and used for nested PCR using the GSP3 primer and the abridged universal amplification primer (AUAP) (5'-GGCCACGCGTCGACTAGTAC-3'). The PCR conditions for nested PCR were the same as the first PCR, except for an annealing temperature of $64^{\circ} \mathrm{C}$.

For 3'-RACE, we used a 3'-Full RACE Core Set kit (Takara) and primers 3F1 (5'-ACCCAGTAGCCACTGAATGTCAC-3') and 3F2 (5'-TGAGCTGCCTACCCTTATCCT AAAG-3'). The locations of the primers are shown in Figure 2. The first-strand cDNA was synthesized at $42^{\circ} \mathrm{C}$ for 60 min using the oligodT-3 adaptor primer and M-MLV reverse transcriptase, and two consecutive PCRs using this cDNA as a template and Ex Taq polymerase (Takara) were carried out as follows: the first PCR was performed under 30 cycles at $94^{\circ} \mathrm{C}$ for $30 \mathrm{~s}, 62^{\circ} \mathrm{C}$ for $30 \mathrm{~s}$, and $72^{\circ} \mathrm{C}$ for $2 \mathrm{~min}$ in a typical PCR mixture with $3 \mathrm{~F} 1$ primer and $3^{\prime} \mathrm{RACE}$ 
Outer Primer (5'-TACCGTCGTTCCACTAGTGATTT-3'). The PCR product was then diluted 1:10 with $\mathrm{ddH}_{2} \mathrm{O}$ and used for nested PCR with the $3 \mathrm{~F} 2$ primer and 3 '-RACE Inner Primer (5'-CGCGGATCCTCCACTAGTGATTTCACTATAGG-3'); the PCRs were the same as mentioned above.

\section{Cloning and DNA sequencing}

The final PCR products were cloned into pMD-18T vector (Takara), and recombinant plasmids were transformed into Escherichia coli competent cells (strain DH5 $\alpha$ ). About 30 positive clones of 5'-RACE and 50 positive clones of 3'-RACE, from the three independent amplifications, respectively, were sequenced. Sequencing was conducted on both strands using the pMD-18T vector primers at the Shanghai Sangon Biological Engineering Technology and Services Co., Ltd., China. Nucleotide sequence alignments, translations, and comparisons were carried out using DNAMAN (version 5.2.10)

\section{Multiple sequence alignment and phylogenetic analyses}

Multiple sequence alignments of tetrapodian FSHB open reading frame (ORF) were performed with the Clustal X program, version 2.0 (Larkin et al., 2007). Values used for pairwise alignments were gap opening penalty 30 and gap extension penalty 0.8 . Values for multiple alignments were gap opening penalty 15 , gap extension penalty 0.3 , and delay divergent sequences $30 \%$. The phylogenetic and molecular evolutionary analysis based on the aligned ORF sequences were constructed by the Molecular Evolution Genetic Analysis software (MEGA, version 4.0) (Kumar et al., 2008). Maximum parsimony, minimum evolution, and neighbor-joining methods with p-distance model for multiple nucleotide substitutions produced very similar topologies, and the tree inferred from the neighbor-joining method is shown. For deriving confidence values of this analysis, bootstrap trials were replicated 1000 times. The GenBank accession numbers and references of selected species used for ORF sequences alignment and phylogenetic analysis in this study are indicated in Table 1.

\section{RESULTS AND DISCUSSION}

Two PCR products, approximately 650 and $1300 \mathrm{bp}$, were obtained from the bovine FSHB cDNA using 5'- and 3'-RACE (Figure 1). The results were repeated twice and confirmed for the three different individuals. The cloned nucleotide sequences were validated to encode the bovine FSHB subunit precursor molecule by comparing with the reported sequences (GenBank Nos. M14853 and NM_174060). Two different full-length cDNAs, 1679 and $1763 \mathrm{bp}$, respectively, resulting from different polyadenylation sites were identified in the wild-type bull pituitary. One canonical polyadenylation signal (AATAAA) was present at $17 \mathrm{bp}$ upstream of the poly (A) tail in the short transcript, and another AATAAA was present at $12 \mathrm{bp}$ upstream of the poly (A) tail in the long transcript (Figure 2). However, we did not find a different alternate splicing site in exon 1 from 29 positive clones of 5'-RACE, which indicated that it may be different from the human FSHB precursor molecules. 
Table 1. Species and references of $F S H B$ s used for ORF sequence alignment and phylogenetic analysis in this study.

\begin{tabular}{|c|c|c|c|c|}
\hline Animal class/species & Scientific name & Order & Accession No. & Reference \\
\hline \multicolumn{5}{|l|}{ Mammals } \\
\hline Cattle & Bos taurus & Cetartiodactyla & GQ163481 & Present study \\
\hline Cattle & Bos taurus & Cetartiodactyla & M14853 & Maurer and Beck, 1986 \\
\hline Buffalo & Bubalus bubalis & Cetartiodactyla & EF710660 & - \\
\hline Deer & Cervus nippon & Cetartiodactyla & AY 156688 & - \\
\hline Sheep & Ovis aries & Cetartiodactyla & X15493 & Mountford et al., 1989 \\
\hline Swine & Sus scrofa & Cetartiodactyla & AF134151 & Li et al., 2000 \\
\hline Horse & Equus caballus & Perissodactyla & AB029157 & Saneyoshi et al., 2001 \\
\hline Tiger & Panthera tigris altaica & Carnivora & AF540937 & - \\
\hline Panda & Ailuropoda melanoleuca & Carnivora & AF448454 & Liao et al., 2003 \\
\hline Human & Homo sapiens & Primates & NM_000510 & Jameson et al., 1988 \\
\hline Squirrel monkey & Saimiri boliviensis boliviensis & Primates & DQ143873 & Scammell et al., 2008 \\
\hline Owl monkey & Aotus nancymaae & Primates & DQ200807 & Scammell et al., 2008 \\
\hline Macaque & Macaca fascicularis & Primates & AJ781395 & Schmidt et al., 1999 \\
\hline Possum & Trichosurus vulpecula & Diprotodontia & AF008550 & Lawrence et al., 1997 \\
\hline Rabbit & Oryctolagus cuniculus & Glires & AY614704 & Noguchi et al., 2006 \\
\hline Rat & Rattus norvegicus & Rodentia & NM 001007597 & Maurer, 1987 \\
\hline Armenian hamster & Cricetulus migratorius & Rodentia & $\mathrm{AB} 235911$ & - \\
\hline Chinese hamster & Cricetulus griseus & Rodentia & AB248599 & - \\
\hline Djungarian hamster & Phodopus sungorus & Rodentia & AB252645 & - \\
\hline Syrian hamster & Mesocricetus auratus & Rodentia & AB241062 & - \\
\hline Mastomys & Mastomys coucha & Rodentia & AY458603 & Takano et al., 2004 \\
\hline Mongolian gerbil & Meriones unguiculatus & Rodentia & AY376457 & Koura et al., 2004 \\
\hline Mouse & Mus musculus & Rodentia & BC061159 & Strausberg et al., 2002 \\
\hline \multicolumn{5}{|l|}{ Birds } \\
\hline Chicken & Gallus gallus & Galliformes & AY029204 & Shen and Yu, 2002 \\
\hline Quail & Coturnix japonica & Galliformes & AB086952 & Kikuchi et al., 1998 \\
\hline Duck & Anas platyrhynchos & Anseriformes & DQ232890 & Shen et al., 2006 \\
\hline Goose & Anser cygnoides & Anseriformes & EU563910 & - \\
\hline \multicolumn{5}{|l|}{ Reptiles } \\
\hline Reeves' turtle & Mauremys reevesii & Testudinoidea & AB085201 & Aizawa and Ishii, 2003 \\
\hline Softshell turtle & Pelodiscus sinensis & Testudinoidea & DQ234263 & Chien et al., 2005 \\
\hline \multicolumn{5}{|l|}{ Amphibians } \\
\hline Toad & Bufo japonicus & Anura & AB085668 & Komoike and Ishii, 2003 \\
\hline Leopard frog & Rana pipiens & Anura & DQ054790 & - \\
\hline
\end{tabular}

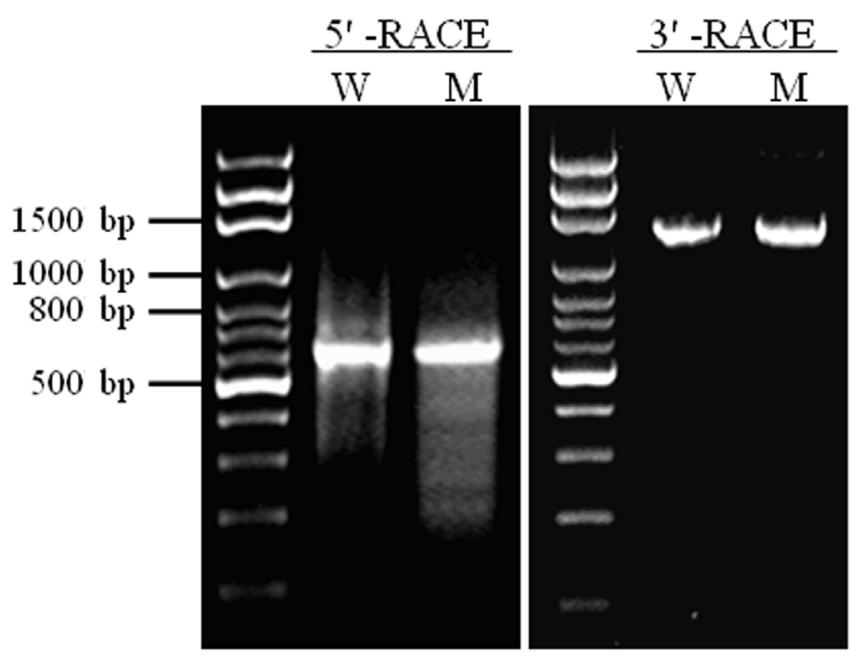

Figure 1. The products of 5'- and 3'-RACE were resolved on 1\% agarose gels, stained with ethidium bromide, and photographed under UV illumination. Lane $W=$ wild-type. Lane $M=$ mutation. 


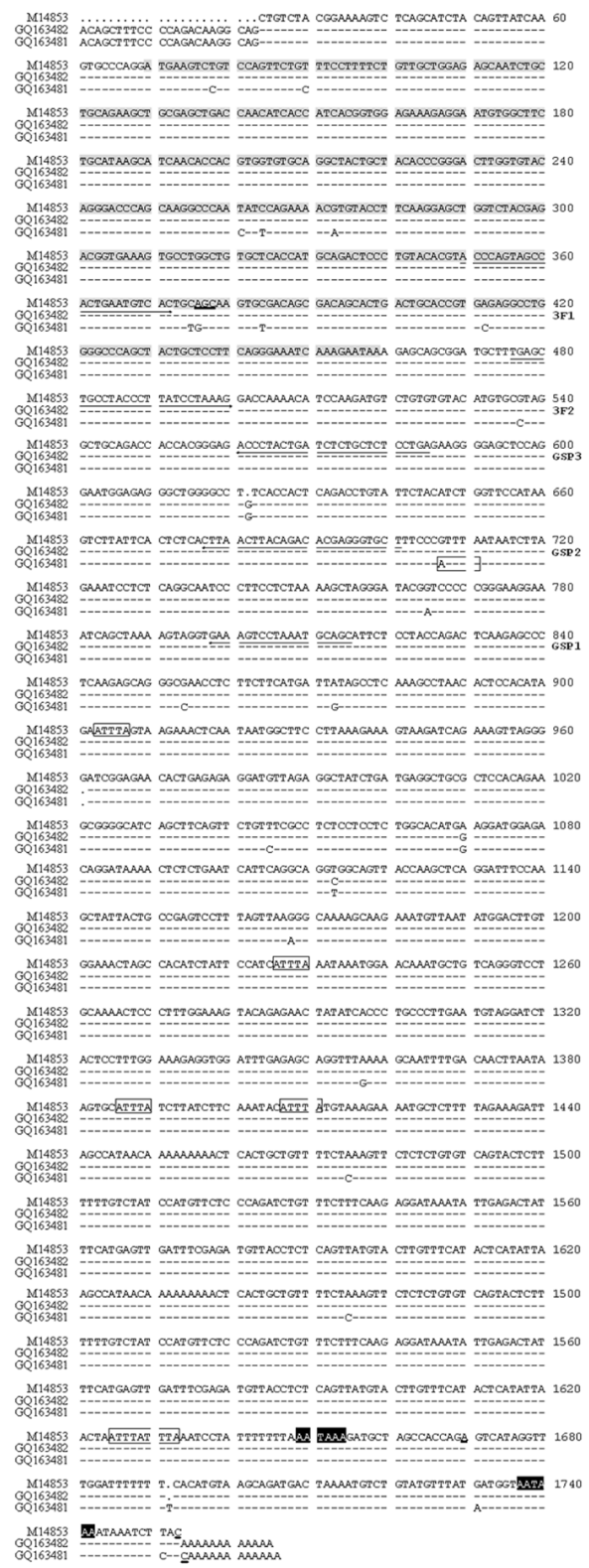

Figure 2. Sequence alignment of the bovine $F S H B$ cDNA comparing that cloned in this study and that previously reported. The open reading frame is shown in a shaded box, the bases missing in M14853 are indicated by dots, and the identical nucleotides are indicated by hyphens. Arrows indicate primer positions (3F1, 3F2, GSP3, GSP2, and GSP1). The substitution 376A $>$ G led to the amino acid replacement Ser84Gly, underlined here. Potential polyadenylational signals are indicated with white letters, and putative polyadenylation sites are underlined. Motifs of AUUUA in the 3'-UTR are boxed. The nucleotide sequences of the bovine FSHB cDNA obtained from this study are available in the GenBank database under accession numbers GQ163481 to GQ163484, and the sequence was the same between Simmental and Charolais breeds. 
Although the two different transcripts could not be validated using Northern blot analysis due to the close length, we could verify and confirm this in the 48 positive clones of 3'-RACE, which included 31 long and 17 short mRNA transcripts, and these two different polyadenylation signal (AATAAA) locations were basically consistent with the reported species such as rabbit (Noguchi et al., 2006) and toad (Komoike and Ishii, 2003). The 3'-UTRs of many mRNAs, especially those of the early responsive genes (ERGs), are involved in posttranscriptional regulation (Pesole et al., 2001; Rabani et al., 2008). It is interesting that among the gonadotropin hormone subunits, only FSHB mRNA contains a unique 3'-UTR, which is highly conserved across the species, but the role of the 3'-UTR in the physiological regulation of FSHB gene expression is still not very clear. The discovery of different transcripts will provide very important information for further study of the role of the longer 3'-UTR in FSHB mRNA.

Four different transcripts were detected combined with the linked mutations with different polyadenylation sites in the mutation-bearing pituitaries. Among them, two different cDNA sequences resulted from 23 linked mutations, including 9 substitutions in the coding region, of which 376A $>$ G led to the amino acid replacement Ser84Gly in the mature protein (Figure 2). As the FSHB cDNA sequences obtained were based on clones derived from PCR amplification, we considered the possibility that some of the sequence results obtained in this study might have been due to PCR or sequencing errors. However, scores of positive clones of one or two independent PCR amplifications from the same bulls were sequenced, and all the sequences reported here were confirmed by sequence alignment for the same individual. Thus, we ascertained that these sequences represent genuine bovine $F S H B$ cDNA sequences and not PCR artifacts or sequencing errors.

Sequence analyses of the rat, mouse, human, ovine, and bovine FSHB 3'-UTR had revealed the presence of elements implicated in mRNA instability and translational control such as AU-rich element (AUUUA, ARE) and lipoxygenase differentiation control elements (Manjithaya and Dighe, 2004). Thirteen substitutions and one-base insertion were found in the FSHB 3'-UTR of mutation-bearing bulls, of which $707 \mathrm{G}>$ A created a new ARE. Thus, there were seven "AUUUA" motifs after six original ARE in such special FSHB mRNA transcript (Figure 2). It is known that ARE can be described as a signal for rapid mRNA degradation (Jarrousse et al., 1999; Zhang et al., 2002; Barreau et al., 2005), so this single nucleotide substitution may be one of the most important reasons for explaining the lower serum FSH concentration in the mutation-bearing individuals. However, further studies are still needed to elucidate the significance and mechanism of the "AUUUA" motif in bovine FSHB expression.

All transcripts had an ORF of $309 \mathrm{bp}$, beginning with the first translation initiation codon at position 70 and ending with the stop codon at position 459, which encoded the same precursor protein. The signal peptide cleavage site was at position 126, yielding a signal peptide of 19 amino acids and a mature peptide of 110 amino acids. The phylogenetic tree of tetrapodian FSHB was presented based on their alignment in ORF sequences (Figure 3). In general, species for the same animal classes are clustered in groups. The homology of two different ORF sequences of the bovine $F S H B$ precursor protein resulting from linked mutations was $97.7 \%$. When the ORF sequences of wild-type and mutation-bearing genes were compared with those of other tetrapodian species, respectively, a closer phylogenetic relationship with buffalo (95.9 and 96.7\%) and ovine (95.4 and 96.7\%) than with deer (92.3 and 93.6\%) and swine (90.8 and 91.8\%) was observed in the Cetartiodactyla. Intermediate identities with the birds and reptiles were about $70 \%$, and the lowest level of homology was found with the 


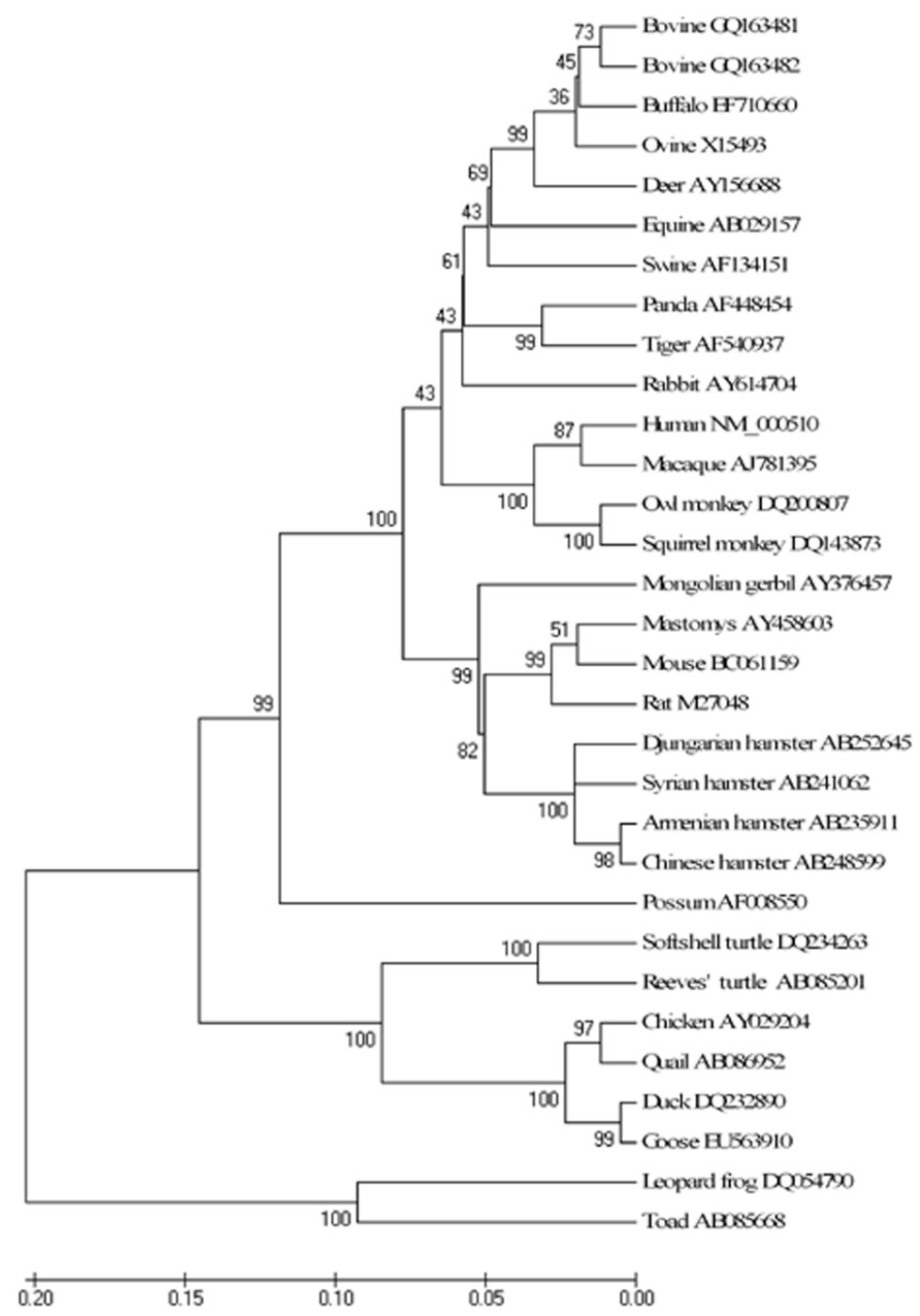

Figure 3. A phylogenetic tree based on the aligned ORF sequences of the tetrapodian FSHBs. GenBank accession numbers and references of $F S H B$ sequences analyzed in this study are indicated in Table 1.

leopard frog (57.8 and 58.1\%). The high genetic diversity of bovine FSHB mRNA implies that it has undergone substantial alteration of nucleotide sequence during the course of evolution, which provided the necessary information for understanding the $F S H B$ evolutionary pattern even though the mutations are a negative change for frozen semen production.

\section{ACKNOWLEDGMENTS}

Research supported by the National Nature Science Foundation (\#30972100), Ear- 
marked Fund for Modern Agro-Industry Technology Research System (\#nycytx-38), and Scientific and Technical Development Program of Jilin Province (\#20090237) of China.

\section{REFERENCES}

Aizawa Y and Ishii S (2003). Cloning of complimentary deoxyribonucleic acid encoding follicle-stimulating hormone and luteinizing hormone beta subunit precursor molecules in Reeves's turtle (Geoclemys reevesii) and Japanese grass lizard (Takydromus tachydromoides). Gen. Comp. Endocrinol. 132: 465-473.

Barreau C, Paillard L and Osborne HB (2005). AU-rich elements and associated factors: are there unifying principles? Nucleic Acids Res. 33: 7138-7150.

Chien JT, Shen ST, Lin YS and Yu JY (2005). Molecular cloning of the cDNA encoding follicle-stimulating hormone beta subunit of the Chinese soft-shell turtle Pelodiscus sinensis, and its gene expression. Gen. Comp. Endocrinol. 141: 190-200.

Dai L, Zhao Z, Zhao R, Xiao S, et al. (2009). Effects of novel single nucleotide polymorphisms of the FSH beta-subunit gene on semen quality and fertility in bulls. Anim. Reprod. Sci. 114: 14-22.

de Kretser DM, Buzzard JJ, Okuma Y, O’Connor AE, et al. (2004). The role of activin, follistatin and inhibin in testicular physiology. Mol. Cell Endocrinol. 225: 57-64.

Dias JA, Cohen BD, Lindau-Shepard B, Nechamen CA, et al. (2002). Molecular, structural, and cellular biology of follitropin and follitropin receptor. Vitam. Horm. 64: 249-322.

Druet T, Fritz S, Sellem E, Basso B, et al. (2009). Estimation of genetic parameters and genome scan for 15 semen characteristics traits of Holstein bulls. J. Anim. Breed. Genet. 126: 269-277.

Geyer CB, Inselman AL, Sunman JA, Bornstein S, et al. (2009). A missense mutation in the Capza 3 gene and disruption of F-actin organization in spermatids of repro32 infertile male mice. Dev. Biol. 330: 142-152.

Gharib SD, Wierman ME, Shupnik MA and Chin WW (1990). Molecular biology of the pituitary gonadotrophins. Endocr. Rev. 11: 177-199.

Jameson JL, Becker CB, Lindell CM and Habener JF (1988). Human follicle-stimulating hormone $\beta$-subunit gene encodes multiple messenger ribonucleic acids. Mol. Endocrinol. 2: 806-815.

Jarrousse AS, Petit F, Kreutzer-Schmid C, Gaedigk R, et al. (1999). Possible involvement of proteasomes (prosomes) in AUUUA-mediated mRNA decay. J. Biol. Chem. 274: 5925-5930.

Kikuchi M, Kobayashi M, Ito T, Kato Y, et al. (1998). Cloning of complementary deoxyribonucleic acid for the folliclestimulating hormone-beta subunit in the Japanese quail. Gen. Comp. Endocrinol. 111: 376-385.

Komoike Y and Ishii S (2003). Cloning of cDNAs encoding the three pituitary glycoprotein hormone beta subunit precursor molecules in the Japanese toad, Bufo japonicus. Gen. Comp. Endocrinol. 132: 333-347.

Koura M, Handa H, Noguchi Y, Takano K, et al. (2004). Sequence analysis of cDNA encoding follicle-stimulating hormone and luteinizing hormone beta-subunits in the Mongolian gerbil (Meriones unguiculatus). Gen. Comp. Endocrinol. 136: 406-410.

Kumar S, Nei M, Dudley J and Tamura K (2008). MEGA: a biologist-centric software for evolutionary analysis of DNA and protein sequences. Brief Bioinform. 9: 299-306.

Kumar TR (2005). What have we learned about gonadotropin function from gonadotropin subunit and receptor knockout mice? Reproduction 130: 293-302.

Larkin MA, Blackshields G, Brown NP, Chenna R, et al. (2007). Clustal W and Clustal X version 2.0. Bioinformatics 23: 2947-2948.

Lawrence SB, Vanmontfort DM, Tisdall DJ, McNatty KP, et al. (1997). The follicle-stimulating hormone beta-subunit gene of the common brushtail possum (Trichosurus vulpecula): analysis of cDNA sequence and expression. Reprod. Fertil. Dev. 9: 795-801.

Li MD, Rohrer GA, Wise TH and Ford JJ (2000). Identification and characterization of a new allele for the beta subunit of follicle-stimulating hormone in Chinese pig breeds. Anim. Genet. 31: 28-30.

Liao MJ, Zhu MY, Zhang ZH, Zhang AJ, et al. (2003). Cloning and sequence analysis of FSH and LH in the giant panda (Ailuropoda melanoleuca). Anim. Reprod. Sci. 77: 107-116.

Lin CL, Jennen DG, Ponsuksili S, Tholen E, et al. (2006). Haplotype analysis of beta-actin gene for its association with sperm quality and boar fertility. J. Anim. Breed. Genet. 123: 384-388.

Manjithaya RR and Dighe RR (2004). The 3' untranslated region of bovine follicle-stimulating hormone beta messenger RNA downregulates reporter expression: involvement of AU-rich elements and transfactors. Biol. Reprod. 71: 1158-1166.

Maurer RA (1987). Molecular cloning and nucleotide sequence analysis of complementary deoxyribonucleic acid for the 
beta-subunit of rat follicle stimulating hormone. Mol. Endocrinol. 1: 717-723.

Maurer RA and Beck A (1986). Isolation and nucleotide sequence analysis of a cloned cDNA encoding the beta-subunit of bovine follicle-stimulating hormone. DNA 5: 363-369.

Mountford PS, Bello PA, Brandon MR and Adams TE (1989). Cloning and DNA sequence analysis of the cDNA for the precursor of ovine follicle stimulating hormone beta-subunit. Nucleic Acids Res. 17: 6391.

Noguchi Y, Takano K, Koura M, Uchio-Yamada K, et al. (2006). Sequence analysis of cDNA encoding rabbit folliclestimulating hormone beta-subunit precursor protein. Gen. Comp. Endocrinol. 147: 231-235.

Pesole G, Mignone F, Gissi C, Grillo G, et al. (2001). Structural and functional features of eukaryotic mRNA untranslated regions. Gene 276: 73-81.

Pierce JG and Parsons TF (1981). Glycoprotein hormones: structure and function. Annu. Rev. Biochem. 50: 465-495.

Rabani M, Kertesz M and Segal E (2008). Computational prediction of RNA structural motifs involved in posttranscriptional regulatory processes. Proc. Natl. Acad. Sci. U. S. A. 105: 14885-14890.

Ren DR, Ren J, Xing YY, Guo YM, et al. (2009). A genome scan for quantitative trait loci affecting male reproductive traits in a White Duroc x Chinese Erhualian resource population. J. Anim. Sci. 87: 17-23.

Saneyoshi T, Min KS, Jing MX, Nambo Y, et al. (2001). Equine follicle-stimulating hormone: molecular cloning of beta subunit and biological role of the asparagine-linked oligosaccharide at asparagine ${ }^{56}$ of alpha subunit. Biol. Reprod. 65: 1686-1690.

Scammell JG, Funkhouser JD, Moyer FS, Gibson SV, et al. (2008). Molecular cloning of pituitary glycoprotein alphasubunit and follicle stimulating hormone and chorionic gonadotropin beta-subunits from New World squirrel monkey and owl monkey. Gen. Comp. Endocrinol. 155: 534-541.

Schmidt A, Gromoll J, Weinbauer GF, Galla HJ, et al. (1999). Cloning and expression of cynomolgus monkey (Macaca fascicularis) gonadotropins luteinizing hormone and follicle-stimulating hormone and identification of two polymorphic sites in the luteinizing hormone beta subunit. Mol. Cell Endocrinol. 156: 73-83.

Shen ST and Yu JY (2002). Cloning and gene expression of a cDNA for the chicken follicle-stimulating hormone (FSH)beta-subunit. Gen. Comp. Endocrinol. 125: 375-386.

Shen ST, Cheng YS, Shen TY and Yu JY (2006). Molecular cloning of follicle-stimulating hormone (FSH)-beta subunit cDNA from duck pituitary. Gen. Comp. Endocrinol. 148: 388-394.

Strausberg RL, Feingold EA, Grouse LH, Derge JG, et al. (2002). Generation and initial analysis of more than 15,000 fulllength human and mouse cDNA sequences. Proc. Natl. Acad. Sci. U. S. A. 99: 16899-16903.

Takano K, Koura M, Noguchi Y, Yamamoto Y, et al. (2004). Sequence analysis of cDNA encoding follicle-stimulating hormone and luteinizing hormone beta-subunits in the Mastomys (Praomys coucha). Gen. Comp. Endocrinol. 138: 281-286.

Wimmers K, Lin CL, Tholen E, Jennen DG, et al. (2005). Polymorphisms in candidate genes as markers for sperm quality and boar fertility. Anim. Genet. 36: 152-155.

Xing Y, Ren J, Ren D, Guo Y, et al. (2009). A whole genome scanning for quantitative trait loci on traits related to sperm quality and ejaculation in pigs. Anim. Reprod. Sci. 114: 210-218.

Zhang T, Kruys V, Huez G and Gueydan C (2002). AU-rich element-mediated translational control: complexity and multiple activities of trans-activating factors. Biochem. Soc. Trans. 30: 952-958. 